\title{
Alimentação de Ctenogobius shufeldti (Jordan e Eigenmann, 1887) (Teleostei, Gobiidae) na Baía de Guaratuba, Atlântico oeste subtropical
}

\author{
Diego Zanlorenzi ${ }^{1}$ \\ Paulo de Tarso Chaves ${ }^{2 *}$ \\ ${ }^{1}$ PPG em Zoologia, Universidade Federal do Paraná, Curitiba - PR, Brasil \\ ${ }^{2}$ Departartamento de Zoologia, Universidade Federal do Paraná \\ Caixa Postal 19020, CEP 81531-980, Curitiba - PR, Brasil \\ * Autor para correspondência \\ ptchaves@ufpr.br
}

Submetido em 15/02/2010

Aceito para publicação em 25/10/2010

\section{Resumo}

A dieta do teleósteo Ctenogobius shufeldti foi estudada na Baía de Guaratuba, litoral sul do Brasil, objetivando descrever variações espaço-temporais e compreender a participação dessa espécie criptobêntica na teia trófica da comunidade deste sistema estuarino. Foram analisados 397 espécimes (comprimento total de 21 a 70mm) em 12 meses de amostragem junto à vegetação marginal da região mais interna da Baía e porção final de dois rios afluentes. O conteúdo estomacal foi identificado e os dados foram transformados em Frequência de Ocorrência e Contagem de Pontos. A conjugação desses dois métodos através do Índice Alimentar informou que a dieta da espécie compõe-se principalmente de Ostracoda $(I A=50,51)$ e Tanaidacea $(I A=40,85)$ somados a algas filamentosas Chlorophyta $(I A=2,15)$ e Rodophyta $(I A=0,15)$ e Material Vegetal Superior $(I A=4,99)$, incluindo secundariamente, Gastropoda $(I A=1,21)$, Amphipoda $(I A=0,10)$, Isopoda $(I A=0,01)$, Cirripedia $(I A=0,01)$, larvas de Decapoda $(I A=0,01)$ e Insecta $(I A=0,01)$. Exceto pelas larvas de Decapoda, os itens consumidos foram os mesmos nos dois rios. A dieta foi mais diversificada nestes que na Baía, área em que Amphipoda e Cirripedia não foram registrados. Algas filamentosas Chlorophyta e Rodophyta não haviam sido reportadas na dieta da espécie em estuários próximos a Guaratuba. Segundo Depczynski e Bellwood (2003), sobre dieta de peixes criptobênticos, conclui-se que na região de estudo C. shufeldti disponibiza energia a outros componentes da teia trófica não apenas pela predação de animais bentônicos, mas também pela ingestão de algas e outros vegetais. Propõe-se então que seu largo espectro trófico, compreendendo invertebrados e vegetais, resulte da influência marinha e continental a que a região estudada se encontra submetida.

Palavras-chave: Dieta, Estuário, Ostracoda, Peixes criptobênticos, Rios, Tanaidacea

\section{Abstract}

Diet of Ctenogobius shufeldti (Jordan and Eigenmann, 1887) (Teleostei, Gobiidae) in Guaratuba Bay, subtropical west Atlantic. The diet composition of the American freshwater goby, Ctenogobius shufeldti, was investigated in an estuarine area and two of its tributaries. 397 individuals, total length from 21 to $70 \mathrm{~mm}$, were sampled over a period of 12 months and their stomach contents were analysed by the Frequency of Occurrence and Number of Points methods. According to the Alimentary Index, diet was constituted mainly by Ostracoda $(\mathrm{IA}=50.51)$ and Tanaidacea $(\mathrm{IA}=40.85)$, and secondarily by Gastropoda $(\mathrm{IA}=1.21)$, Amphipoda $(\mathrm{IA}=0.10)$, 
Isopoda (IA=0.01), Cirripedia (IA=0.01), Decapoda larvae (IA=0.01), Insecta (IA=0.01), Chlorophyta $(I A=2.15)$ and Rodophyta $(\mathrm{IA}=0.15)$ filamentous algae, and plants $(\mathrm{IA}=4.99)$. Diversity of diet was higher in rivers than in the Bay. Except for Decapoda larvae, items found in the two rivers were the same. In the Bay, Amphipoda and Cirripedia were not found. Previous to this research, the filamentous algae Chlorophyta and Rodophyta had not been noted in the diet of this species in estuaries close to Guaratuba. In view of this, and regarding the possible roles played by cryptobenthic fish species in aquatic communities (Depczynski and Bellwood, 2003), it is concluded that $C$. shufeldti takes part in trophic dynamics feeding on benthic organisms as well as on algae, a behaviour that can result from both marine and continental influences existing in the region.

Key words: Cryptobenthic fish, Estuary, Feeding habits, Ostracoda, Rivers, Tanaidacea

\section{Introdução}

Conhecidos como "emborês" ou "amborês", os peixes da família Gobiidae reúnem aproximadamente 2000 espécies e mais de 200 gêneros, constituindo a maior família de peixes tropicais marinhos do mundo. O corpo em geral é curto, com comprimento inferior a $10 \mathrm{~cm}$, a cabeça é larga, os olhos situam-se em posição superior, e as nadadeiras pélvicas unem-se formando um disco. Esses peixes são na maioria marinhos (NELSON, 2006), e parte das espécies distribuídas no Sudeste-Sul do Brasil habitam áreas estuarinas de manguezal (FIGUEIREDO; MENEZES, 1985). Exceto pelo interesse comercial na aquariofilia ou como isca na atividade de pesca, as espécies da família não apresentam valor econômico direto para o homem. Em geral, os peixes da família Gobiidae são criptobênticos (FROESE e PAULY, 2010), os quais são definidos como aqueles cujos adultos são, visual e/ou comportamentalmente, crípticos, não visíveis de cima, e que mantêm estreita associação com o ambiente bentônico (PATZNER, 1999; DEPCZYNSKI; BELLWOOD, 2003).

Na Baía de Guaratuba, litoral do Estado do Paraná, ocorrem ao menos oito espécies da família: Bathygobius soporator (Valenciennes, 1837), Ctenogobius shufeldti (Jordan; Eigenmann, 1887), C. smaragdus (Valenciennes, 1837), C. stigmaticus (Poey, 1860), Gobioides broussonnetii Lacepède, 1800, Gobionellus oceanicus (Pallas, 1770), Gobionellus stomatus Starks, 1913 e Microgobius meeki Evermann \& Marsh, 1899 (CHAVES; CORRÊA, 1998; CHAVES; VENDEL, 2001). Peixes desta família apresentam um espectro alimentar muito variado, composto, conforme a espécie, de uma grande diversidade de invertebrados e vegetais (ex.: BOUCHEREAU et al., 1991; JOYEUX et al., 1991; ELGUEZABAL et al., 2003; CABERTY et al., 2004). De acordo com a revisão de Chaves e Bouchereau (2004), atualizada por Chaves e Vendel (2008), o conhecimento sobre alimentação de peixes na Baía de Guaratuba contempla mais de uma dezena de espécies de teleósteos, entretanto nenhuma pertencente à família Gobiidae.

Segundo Depczynski e Bellwood (2003), embora comumente assuma-se que peixes criptobênticos transfiram energia no sistema apenas nas vias de predação (ex.: predando crustáceos ou sendo predados por animais piscívoros), resultados obtidos em ambientes recifais mostram que eles podem também ser detritívoros, atuando, assim, diretamente na reciclagem de nutrientes do substrato. . Portanto, o presente trabalho tem como objetivo descrever a dieta de $C$. shufeldti na Baía de Guaratuba, bem como suas possíveis variações espaço-temporais, além de avaliar se esta espécie integra a teia trófica da região na primeira das situações acima relatadas (relações presa-predador) ou se na segunda (base detritívora para a teia trófica).

\section{Material e Métodos}

\section{Área de estudo}

A Baía de Guaratuba (2552'S; 48³9'O) é o segundo maior sistema estuarino do litoral do Estado do Paraná, sul do Brasil. Comunica-se com o mar por uma abertura de aproximadamente $500 \mathrm{~m}$ e prolonga-se continente adentro por cerca de $15 \mathrm{~km}$ no sentido leste-oeste, com largura máxima de $5 \mathrm{~km}$ na direção norte-sul. Na extremidade oeste da Baía desembocam seus dois maiores afluentes, os rios Cubatão e São João, ambos procedentes das montanhas vizinhas. Segundo levantamento realizado por Bouchereau e Chaves (2003) num transecto lesteoeste ao longo de um ano (1999), na Baía a temperatura média da água variou de 17 a $27^{\circ} \mathrm{C}$ na superfície e de 20 
a $27^{\circ} \mathrm{C}$ no fundo, sem diferenças significativas ao longo do transecto. A salinidade apresentou valores médios de 2,0 na superfície e 6,5 no fundo, todavia, sendo significativamente menor na extremidade oeste da Baía que próximo ao mar. $\mathrm{O}$ mesmo ocorreu com o $\mathrm{pH}$, o qual apresentou valores médios de 6,9 na superfície e 7,1 no fundo, e também foram significativamente menores na extremidade oeste da Baía que na região próxima ao mar. Portanto, conclui-se que a região de estudo é influenciada tanto pelas massas d'água vindas do oceano como por eventos de origem continental, notadamente as fortes precipitações comuns no verão.

Devido à heterogeneidade espacial das condições abióticas (BOUCHEREAU; CHAVES, 2003), que se reflete também sobre a composição vegetacional das margens (CHAVES; VENDEL, 2008), três áreas de coleta foram definidas: Cubatão e São João, situadas na porção final dos rios homônimos, e Baía, situada na extremidade oeste da Baía de Guaratuba (Figura 1). Todas elas integram uma Área de Proteção Ambiental,

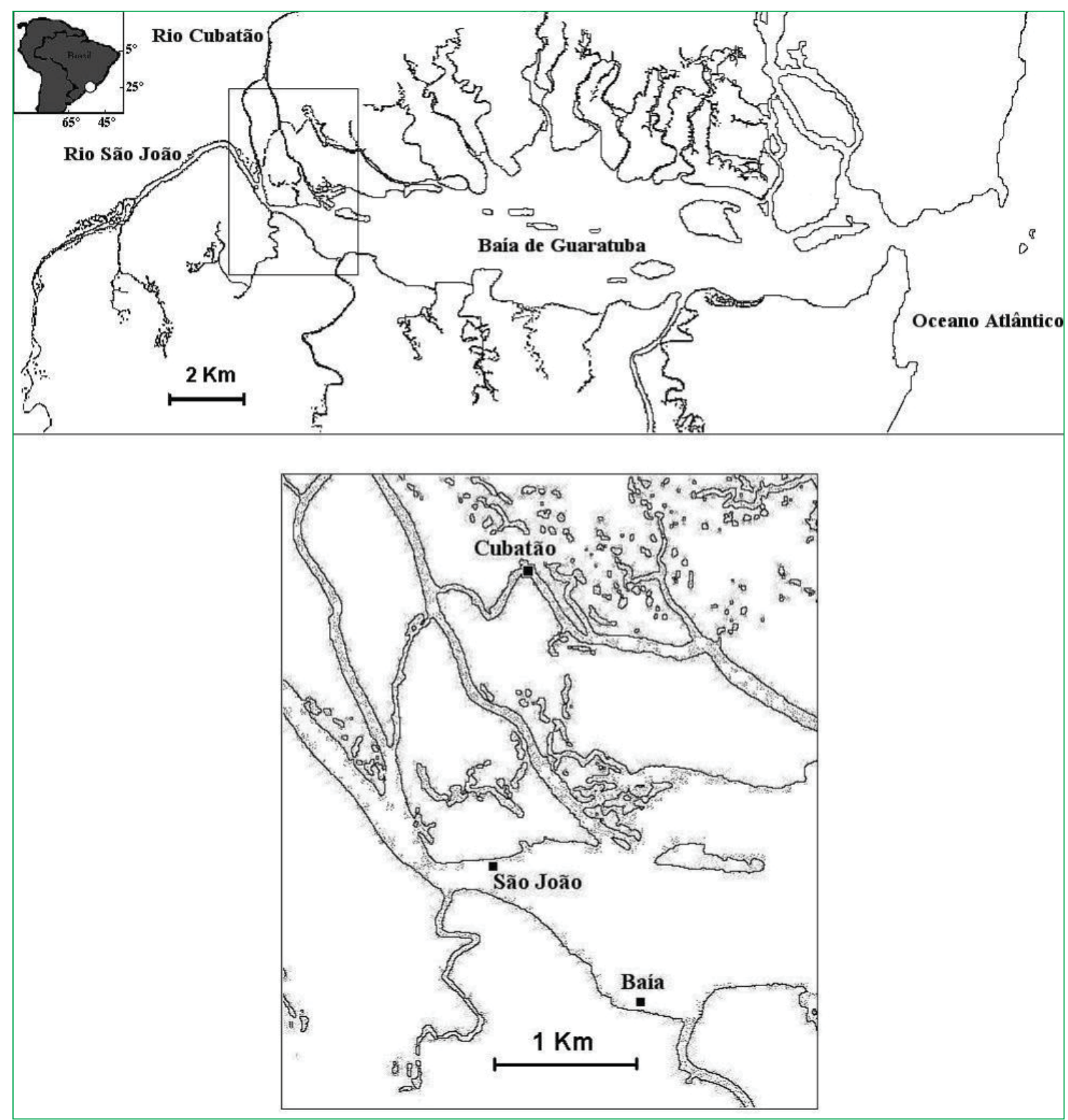

FIGURA 1: Localização da Baía de Guaratuba no litoral sul do Brasil, indicando a zona montante da Baía (retângulo no quadro superior) e as três áreas amostradas (quadro inferior). 
mesmo assim, devido a empreendimentos agrícolas e de mineração implantados a montante, o rio São João está submetido a evidentes impactos antrópicos. Cubatão e Baía apresentam vegetação marginal conhecida como cebolana, Crinum salsum Ravenna (Amaryllidaceae). No São João predomina o piri, Scirpus californicus (MEY, C. A.) Steud (Cyperaceae). Cebolama e piri são parcialmente submersas sob maré baixa. Atrás da vegetação marginal, Baía e Cubatão conservam espécies típicas de manguezal: mangue-vermelho, Rhizophora mangle (Rhizophoraceae), mangue-branco, Laguncularia racemosa (Combretacea) e mangue-preto ou siriúba, Avicennia schaueriana Stapf \& Leechm. Nas três áreas é abundante a gramínea Spartina alterniflora Loisel (Poacea), conhecida como praturá.

\section{Amostragem}

Os espécimes foram capturados mensalmente, de setembro de 2007 a agosto de 2008, sempre no período da manhã, durante a baixamar de marés de quadratura. Em cada ponto, realizou-se medição dos parâmetros físicoquímicos da água: temperatura e salinidade da superfície da água, $\mathrm{pH}$ e transparência. Posteriormente, foram realizados em cada ponto três arrastos de praia, utilizandose rede do tipo picaré, com $22 \mathrm{~m}$ de comprimento, $1,5 \mathrm{~m}$ de altura e abertura de malha $5 \mathrm{~mm}$ entre nós opostos. Os espécimes foram armazenados em recipiente resfriado com gelo e transportados ao laboratório. Devido a dificuldades operacionais, no mês de fevereiro não foi possível amostragem no ponto Cubatão.

\section{Processamento das amostras e análi- se dos dados}

Os espécimes foram medidos (comprimento totalCT; ao nível do mm mais próximo) e fixados em solução de formalina $10 \%$ durante $48 \mathrm{~h}$. Transcorrido esse tempo, foram transferidos e preservados em álcool $70 \%$, até que se realizasse a triagem do conteúdo estomacal.

De um total de 1066 espécimes coletados, 397 $(21 \mathrm{~mm} \leq \mathrm{CT} \leq 70 \mathrm{~mm})$ (Figura 2$)$ apresentavam no estômago pelo menos um item identificável. Os itens foram agrupados em 11 categorias alimentares: Amphipoda, Cirripedia, Chlorophyta Filamentosa
Cenocítica, Gastropoda, Insecta, Isopoda, Larva de Decapoda, Material Vegetal Superior, Ostracoda, Rodophyta Filamentosa e Tanaidacea. Os itens foram quantificados com o uso de dois métodos: 1) Frequência de Ocorrência

$$
F O_{i}=N_{i} / N \times 100
$$

sendo $N_{i}$ o número de estômagos que contêm o item " $i$ " e $N$ o número de estômagos com conteúdo identificável; e 2) Contagem de Pontos, baseado na quantidade de quadrículas ocupada por determinado item alimentar disposto sobre papel milimetrado, onde cada item recebe a pontuação proporcional à área ocupada pelo conjunto de itens

$$
C P_{i}=q_{i} / q \times 100
$$

sendo $\mathrm{q}_{i} \mathrm{o}$ número de quadrículas ocupadas pelo item “” e q o número de quadrículas do conteúdo total. Finalmente, os métodos Frequência de Ocorrência e Contagem de Pontos foram conjugados através do Índice Alimentar (modificado de KAWAKAMI; VAZZOLER, 1980)

$$
I A_{i}=100 \times\left(F O_{i} \times C P_{i}\right) / \sum_{i=1}^{n}\left(F O_{i} \times C P_{i}\right)
$$

sendo n o total de itens “" identificados no conteúdo estomacal da espécie em questão.

$\mathrm{Na}$ análise dos dados e na apresentação dos resultados, os doze meses foram agrupados em estações do ano. Para verificação de similaridades na composição tanto sazonal quanto espacial da dieta, realizou-se análises de agrupamento a partir dos valores de IA transformados por raiz quadrada, utilizando o Índice de Similaridade de Bray-Curtis.

\section{Resultados}

A temperatura da superfície da água variou de 16,1 a $28,0{ }^{\circ} \mathrm{C}$, com os maiores valores registrados no verão e os menores no fim do outono, sendo superior na Baía em relação às demais áreas, exceto no mês de setembro, quando o Cubatão apresentou maior 
temperatura. A salinidade esteve mais elevada de maio a outubro, coincidindo com os meses de outono-inverno, apresentando a maior amplitude na Baía (0 a 15), sendo 0 em todos os pontos do final da primavera ao início do outono. A transparência Secchi seguiu a mesma tendência da salinidade, indicando um aporte marinho no inverno, água mais transparente, e fluvial no verão, água mais turva devido à maior pluviosidade nesta estação. $\mathrm{O}$ pH variou de 5,35 a 8,10, mas não mostrou uma tendência sazonal, tampouco esteve relacionado ao ponto amostral (Figura 2).
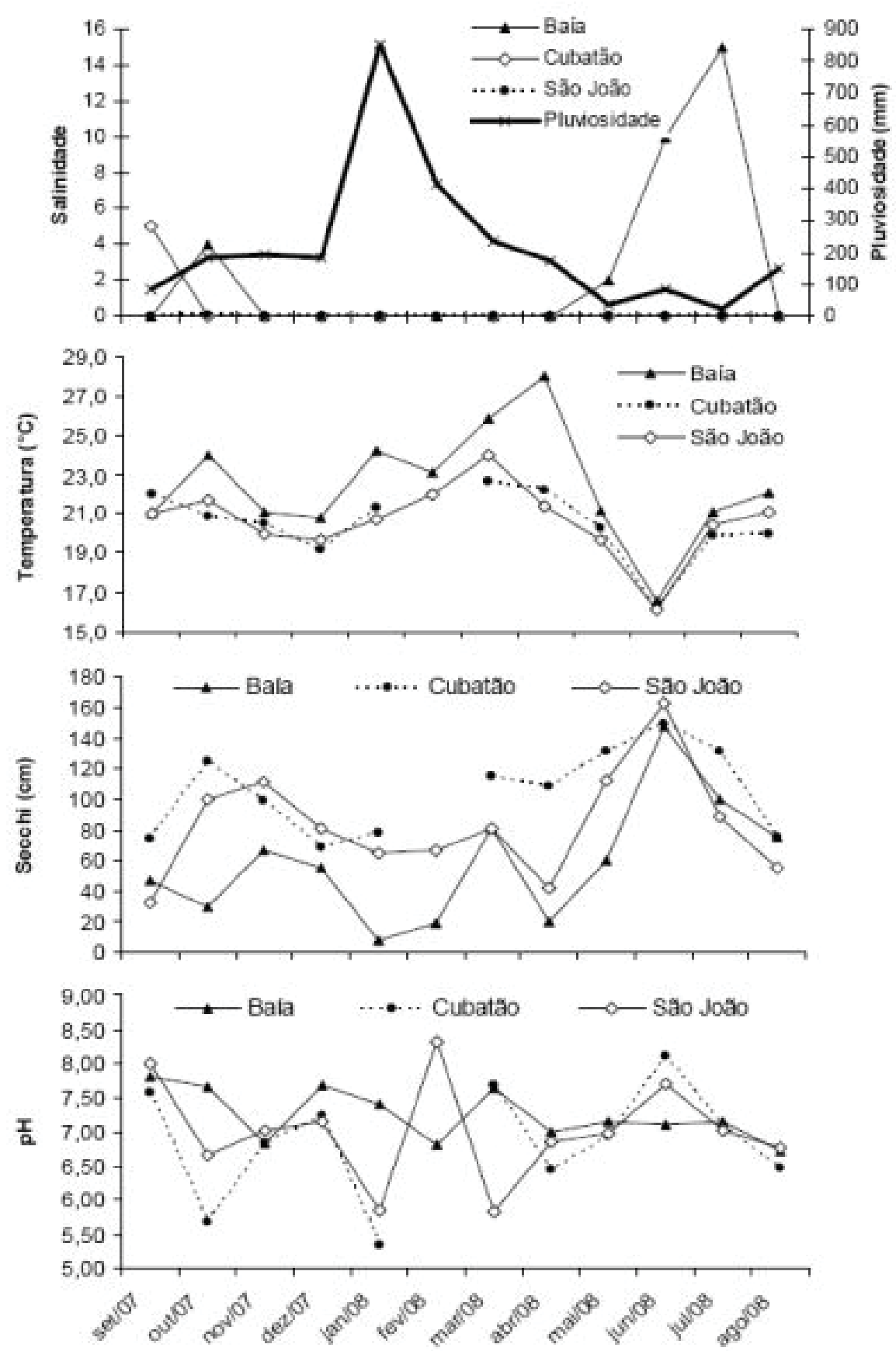

FIGURA 2: Distribuição dos valores de pluviosidade mensal acumulada para a Baía de Guaratuba (Fonte: SIMEPAR), salinidade, temperatura superficial, transparência Secchi e pH segundo o mês e a área de coleta. 
A dieta de $C$. shufeldti compõe-se principalmente de Ostracoda $(I A=50,51)$ e Tanaidacea $(I A=40,85)$ e inclui, secundariamente, Gastropoda $(I A=1,21)$, Amphipoda $(I A=0,10)$, Isopoda $(I A=0,10)$, Cirripedia $(I A=0,10)$, Larvas de Decapoda $(I A=0,10)$, Insecta $(I A=0,10)$, algas filamentosas Chlorophyta $(I A=2,15)$ e Rodophyta $(I A=0,15)$, e Material Vegetal Superior $(I A=4,99)$ (Tabela 1). A dieta apresentou variação sazonal, sendo que na primavera, $32 \mathrm{~mm} \leq \mathrm{CT} \leq 70 \mathrm{~mm}$ (Figura 3 ), houve maior consumo de Ostracoda, seguidos de Tanaidacea e algas nos dois rios, e de Gastropoda na Baía (Tabela 2). No verão, $21 \mathrm{~mm} \leq \mathrm{CT} \leq 67 \mathrm{~mm}$ (Figura 3 ), predominaram Ostracoda no Cubatão e na Baía, e Tanaidacea no São João. Foram itens secundários algas e material vegetal no Cubatão e na Baía, e Ostracoda e algas no São João (Tabela 3). No outono, $30 \mathrm{~mm} \leq \mathrm{CT} \leq 57 \mathrm{~mm}$ (Figura 3 ), Ostracoda foi a categoria mais representativa apenas no Cubatão, enquanto Tanaidacea predominou na Baía e no São João (Tabela 4). Nessa época destacaram-se ainda Gastropoda, material vegetal e Tanaidacea no Cubatão, Ostracoda na Baía e no São João, e algas filamentosas no São João. O inverno, $27 \mathrm{~mm} \leq \mathrm{CT} \leq 68 \mathrm{~mm}$ (Figura 3), foi semelhante ao verão: os Ostracoda predominaram no Cubatão e na Baía, Tanaidacea no São João, e a esses dois itens seguiram-se algas e material vegetal nos dois rios e Gastropoda na Baía (Tabela 5).

TABELA 1: Valores de Frequência de Ocorrência $(F O)$, Contagem de Pontos $(C P)$ e Índice Alimentar $(I A)$ dos itens registrados no conteúdo estomacal de C. shufeldti. CFC: Chlorophyta filamentosa cenocítica; RF: Rodophyta filamentosa; MV: material vegetal. ( ): Comprimentos mínimo e máximo dos " $\mathrm{n}$ " espécimes analisados.

\begin{tabular}{cccc}
\hline & \multicolumn{3}{c}{$\mathrm{N}=397(21-70 \mathrm{~mm})$} \\
\hline Item & FO & CP & IA \\
Ostracoda & 70,78 & 36,78 & 50,51 \\
Tanaidacea & 49,87 & 42,22 & 40,85 \\
Gastropoda & 22,17 & 2,81 & 1,21 \\
Isopoda & 1,76 & 0,33 & 0,01 \\
Amphipoda & 2,77 & 1,86 & 0,10 \\
Cirripedia & 0,50 & 0,10 & 0,01 \\
Insecta & 1,01 & 0,38 & 0,01 \\
LD & 0,50 & 0,28 & 0,01 \\
CFC & 16,37 & 6,78 & 2,15 \\
RF & 8,06 & 1,05 & 0,15 \\
MV & 34,76 & 7,41 & 4,99 \\
\hline TOTAL & - & 100,00 & 100,00 \\
\hline
\end{tabular}

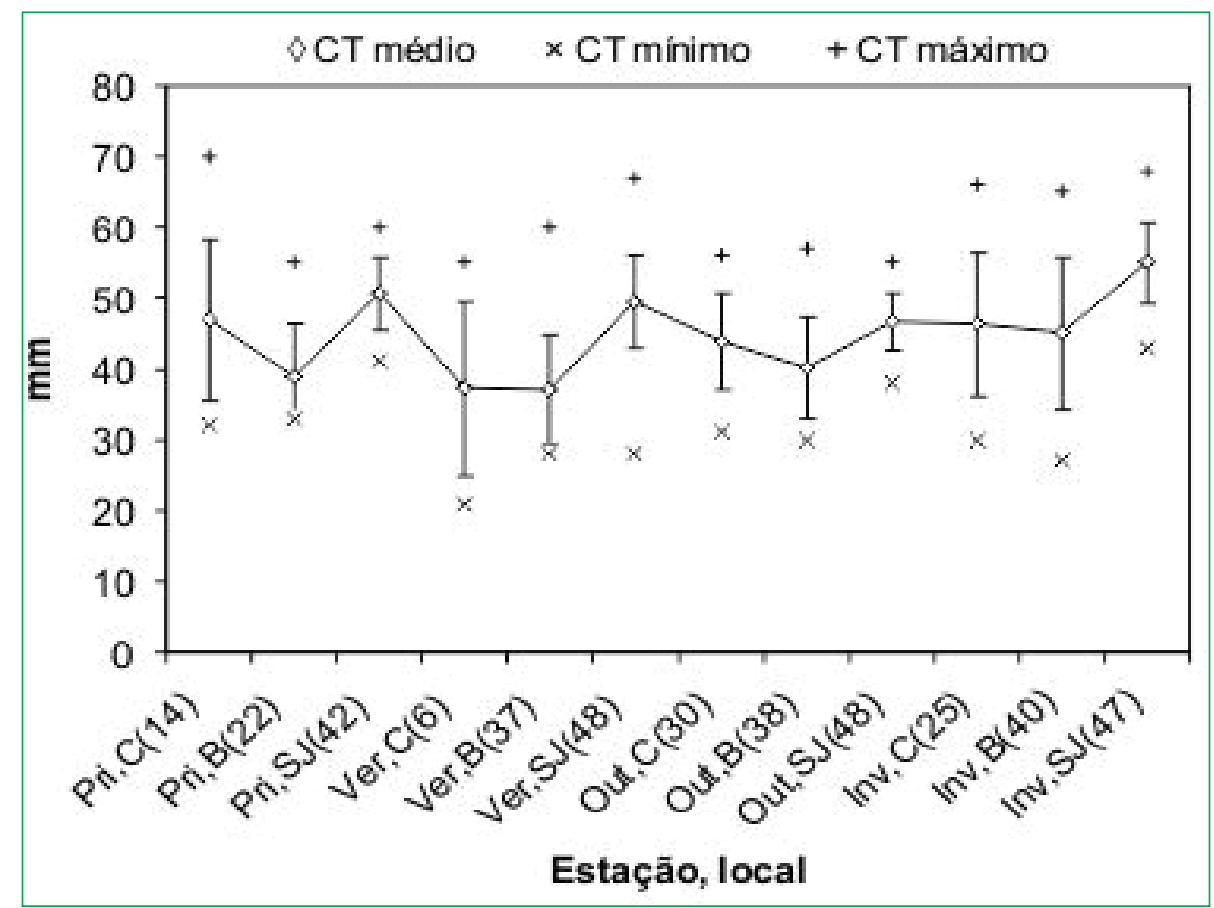

FIGURA 3: Distribuição dos valores médios e desvios-padrões do comprimento total (CT), comprimento máximo e mínimo dos exemplares analisados por estação do ano (primavera, verão, outono, inverno) e área de coleta: Cubatão (C), Baía (B) e São João (SJ). Entre ( ): tamanho da amostra. 
TABELA 2: Valores de Frequência de Ocorrência $(F O)$, Contagem de Pontos $(C P)$ e Índice Alimentar $(I A)$ dos itens registrados no conteúdo estomacal de C. shufeldti nas áreas Cubatão, Baía e São João na primavera. CFC: Chlorophyta filamentosa cenocítica; RF: Rodophyta filamentosa; MV: material vegetal. ( ): Comprimentos mínimo e máximo dos "n" espécimes analisados.

\begin{tabular}{cccccccccc} 
& \multicolumn{3}{c}{$\begin{array}{c}\text { Cubatão } \\
\text { N=14 (32-70mm) }\end{array}$} & \multicolumn{3}{c}{$\begin{array}{c}\text { Baía } \\
\text { N=22 (33-55mm) }\end{array}$} & \multicolumn{3}{c}{$\begin{array}{c}\text { São João } \\
\text { N=42 (41-60mm) }\end{array}$} \\
\hline Item & $\boldsymbol{F O}$ & $\boldsymbol{C P}$ & $\boldsymbol{I} \boldsymbol{A}$ & $\boldsymbol{F O}$ & $\boldsymbol{C P}$ & $\boldsymbol{I} \boldsymbol{A}$ & $\boldsymbol{F O}$ & $\boldsymbol{C P}$ & $\boldsymbol{I} \boldsymbol{A}$ \\
Ostracoda & 78,57 & 61,81 & $\mathbf{6 7 , 9 9}$ & 100,00 & 92,69 & $\mathbf{9 3 , 3 8}$ & 76,19 & 43,95 & $\mathbf{6 5 , 0 5}$ \\
Tanaidacea & 64,29 & 33,60 & $\mathbf{3 0 , 2 6}$ & 31,82 & 4,15 & $\mathbf{1 , 3 9}$ & 50,00 & 34,08 & $\mathbf{3 3 , 1 0}$ \\
Gastropoda & 21,43 & 0,40 & $\mathbf{0 , 1 4}$ & 40,91 & 2,77 & $\mathbf{1 , 1 9}$ & 0,00 & 0,00 & - \\
Isopoda & 0,00 & 0,00 & - & 0,00 & 0,00 & - & 2,38 & 0,15 & $\mathbf{0 , 0 1}$ \\
Amphipoda & 0,00 & 0,00 & - & 0,00 & 0,00 & - & 2,38 & 1,37 & $\mathbf{0 , 0 1}$ \\
CFC & 7,14 & 1,49 & $\mathbf{0 , 1 7}$ & 0,00 & 0,00 & - & 14,29 & 4,19 & $\mathbf{1 , 1 6}$ \\
RF & 42,86 & 1,35 & $\mathbf{0 , 8 3}$ & 0,00 & 0,00 & - & 19,05 & 1,68 & $\mathbf{0 , 6 2}$ \\
MV & 28,57 & 1,35 & $\mathbf{0 , 5 3}$ & 9,09 & 0,39 & $\mathbf{0 , 0 4}$ & 0,00 & 0,00 & - \\
\hline TOTAL & - & 100,00 & $\mathbf{1 0 0 , 0 0}$ & - & 100,00 & $\mathbf{1 0 0 , 0 0}$ & - & 100,00 & $\mathbf{1 0 0 , 0 0}$ \\
\hline
\end{tabular}

TABELA 3: Valores de Frequência de Ocorrência $(F O)$, Contagem de Pontos $(C P)$ e Índice Alimentar $(I A)$ dos itens registrados no conteúdo estomacal de C. shufeldti nas áreas Cubatão, Baía e São João no verão. CFC: Chlorophyta filamentosa cenocítica; RF: Rodophyta filamentosa; MV: material vegetal. ( ): Comprimentos mínimo e máximo dos "n" espécimes analisados.

\begin{tabular}{cccccccccc}
\hline & \multicolumn{3}{c}{$\begin{array}{c}\text { Cubatão } \\
\text { N=6 (21-55mm) }\end{array}$} & \multicolumn{3}{c}{$\begin{array}{c}\text { Baía } \\
\mathbf{N}=\mathbf{3 7}(\mathbf{2 8}-\mathbf{6 0 m m})\end{array}$} & \multicolumn{3}{c}{$\begin{array}{c}\text { São João } \\
\text { N=48 (28-67mm) }\end{array}$} \\
\hline Item & $\boldsymbol{F O}$ & $\boldsymbol{C P}$ & $\boldsymbol{I} \boldsymbol{A}$ & $\boldsymbol{F O}$ & $\boldsymbol{C P}$ & $\boldsymbol{I} \boldsymbol{A}$ & $\boldsymbol{F O}$ & $\boldsymbol{C P}$ & $\boldsymbol{I} \boldsymbol{A}$ \\
Ostracoda & 83,33 & 78,00 & $\mathbf{9 1 , 1 2}$ & 81,08 & 81,03 & $\mathbf{9 4 , 9 6}$ & 56,25 & 5,67 & $\mathbf{4 , 6 4}$ \\
Tanaidacea & 0,00 & 0,00 & - & 10,81 & 4,61 & $\mathbf{0 , 7 2}$ & 79,17 & 76,53 & $\mathbf{8 8 , 2 2}$ \\
Gastropoda & 0,00 & 0,00 & - & 16,22 & 1,63 & $\mathbf{0 , 3 6}$ & 0,00 & 0,00 & - \\
Isopoda & 0,00 & 0,00 & - & 0,00 & 0,00 & - & 4,17 & 0,52 & $\mathbf{0 , 0 3}$ \\
Amphipoda & 0,00 & 0,00 & - & 0,00 & 0,00 & - & 4,17 & 2,01 & $\mathbf{0 , 1 2}$ \\
Insecta & 0,00 & 0,00 & - & 2,70 & 1,90 & $\mathbf{0 , 0 7}$ & 0,00 & 0,00 & - \\
CFC & 0,00 & 0,00 & - & 5,40 & 1,36 & $\mathbf{0 , 1 0}$ & 35,42 & 9,07 & $\mathbf{4 , 6 8}$ \\
RF & 33,33 & 16,00 & $\mathbf{7 , 4 8}$ & 13,51 & 1,36 & $\mathbf{0 , 2 7}$ & 4,17 & 1,66 & $\mathbf{0 , 1 0}$ \\
MV & 16,67 & 6,00 & $\mathbf{1 , 4 0}$ & 29,73 & 8,13 & $\mathbf{3 , 4 9}$ & 33,33 & 4,54 & $\mathbf{2 , 2 0}$ \\
\hline TOTAL & - & 100,00 & $\mathbf{1 0 0 , 0 0}$ & - & 100,00 & $\mathbf{1 0 0 , 0 0}$ & - & 100,00 & $\mathbf{1 0 0 , 0 0}$ \\
\hline
\end{tabular}

TABELA 4: Valores de Frequência de Ocorrência $(F O)$, Contagem de Pontos $(C P)$ e Índice Alimentar $(I A)$ dos itens registrados no conteúdo estomacal de $C$. shufeldti nas áreas Cubatão, Baía e São João no outono. CFC: Chlorophyta filamentosa cenocítica; RF: Rodophyta filamentosa; MV: material vegetal. ( ): Comprimentos mínimo e máximo dos " $n$ " espécimes analisados.

\begin{tabular}{cccccccccc}
\hline & \multicolumn{3}{c}{$\begin{array}{c}\text { Cubatão } \\
\text { N=30 (31-56mm) }\end{array}$} & \multicolumn{3}{c}{$\begin{array}{c}\text { Baía } \\
\mathbf{N}=\mathbf{3 8}(\mathbf{3 0 - 5 7 m m})\end{array}$} & \multicolumn{3}{c}{$\begin{array}{c}\text { São João } \\
\text { N=48 (38-55mm) }\end{array}$} \\
\hline Item & $\boldsymbol{F O}$ & $\boldsymbol{C P}$ & $\boldsymbol{I A}$ & $\boldsymbol{F O}$ & $\boldsymbol{C P}$ & $\boldsymbol{I} \boldsymbol{A}$ & $\boldsymbol{F O}$ & $\boldsymbol{C P}$ & $\boldsymbol{I} \boldsymbol{A}$ \\
Ostracoda & 93,33 & 36,84 & $\mathbf{5 1 , 4 4}$ & 71,05 & 16,36 & $\mathbf{2 3 , 0 2}$ & 50,00 & 11,06 & $\mathbf{1 3 , 4 4}$ \\
Tanaidacea & 36,67 & 23,51 & $\mathbf{1 2 , 9 0}$ & 52,83 & 85,82 & $\mathbf{8 8 , 5 9}$ & 52,08 & 55,04 & $\mathbf{7 0 , 4 4}$ \\
Gastropoda & 73,33 & 16,84 & $\mathbf{1 8 , 4 7}$ & 34,21 & 9,45 & $\mathbf{8 , 4 0}$ & 14,58 & 3,78 & $\mathbf{1 , 3 4}$ \\
Isopoda & 3,33 & 0,70 & $\mathbf{0 , 0 3}$ & 0,00 & 0,00 & - & 0,00 & 0,00 & - \\
Amphipoda & 3,33 & 1,05 & $\mathbf{0 , 0 5}$ & 0,00 & 0,00 & - & 2,08 & 2,19 & $\mathbf{0 , 1 1}$ \\
Insecta & 3,33 & 2,81 & $\mathbf{0 , 1 4}$ & 0,00 & 0,00 & - & 4,17 & 1,89 & $\mathbf{0 , 1 9}$ \\
CFC & 0,00 & 0,00 & - & 13,26 & 4,73 & $\mathbf{1 , 2 3}$ & 22,92 & 16,76 & $\mathbf{9 , 3 4}$ \\
RF & 3,33 & 0,35 & $\mathbf{0 , 0 2}$ & 0,00 & 0,00 & $\mathbf{0 , 0 0}$ & 14,58 & 1,89 & $\mathbf{0 , 6 7}$ \\
MV & 63,33 & 17,89 & $\mathbf{1 6 , 9 5}$ & 10,53 & 3,64 & $\mathbf{0 , 7 8}$ & 27,08 & 6,78 & $\mathbf{4 , 4 6}$ \\
\hline TOTAL & - & 100,00 & $\mathbf{1 0 0 , 0 0}$ & - & 100,00 & $\mathbf{1 0 0 , 0 0}$ & - & 100,00 & $\mathbf{1 0 0 , 0 0}$ \\
\hline
\end{tabular}


TABELA 5: Valores de Frequência de Ocorrência $(F O)$, Contagem de Pontos $(C P)$ e Índice Alimentar $(I A)$ dos itens registrados no conteúdo estomacal de $C$. shufeldti nas áreas Cubatão, Baía e São João no inverno. LD: larva de Decapoda; CFC: Chlorophyta filamentosa cenocítica; RF: Rodophyta filamentosa; MV: material vegetal. ( ): Comprimentos mínimo e máximo dos "n" espécimes analisados.

\begin{tabular}{|c|c|c|c|c|c|c|c|c|c|}
\hline \multirow[b]{2}{*}{ Item } & \multicolumn{3}{|c|}{$\begin{array}{c}\text { Cubatão } \\
\mathrm{N}=25(30-66 \mathrm{~mm})\end{array}$} & \multicolumn{3}{|c|}{$\begin{array}{c}\text { Baía } \\
\mathrm{N}=40(27-65 \mathrm{~mm})\end{array}$} & \multicolumn{3}{|c|}{$\begin{array}{c}\text { São João } \\
\mathrm{N}=47 \text { (43-68mm) }\end{array}$} \\
\hline & $F O$ & $C P$ & $I A$ & $F O$ & $C P$ & $I A$ & $F O$ & $C P$ & $I A$ \\
\hline Ostracoda & 76,00 & 44,91 & 47,91 & 70,00 & 48,68 & 71,00 & 59,57 & 8,08 & 7,82 \\
\hline Tanaidacea & 80,00 & 34,20 & 38,40 & 20,00 & 16,45 & 6,85 & 76,60 & 56,31 & 70,03 \\
\hline Gastropoda & 20,00 & 1,57 & 0,44 & 50,00 & 15,46 & 16,11 & 8,51 & 0,44 & 0,06 \\
\hline Isopoda & 4,00 & 1,57 & 0,09 & 2,50 & 0,33 & $\mathbf{0 , 0 2}$ & 2,13 & 0,44 & $\mathbf{0 , 0 2}$ \\
\hline Amphipoda & 0,00 & 0,00 & - & 0,00 & 0,00 & - & 12,77 & 7,41 & 1,54 \\
\hline Cirripedia & 4,00 & 0,26 & 0,01 & 0,00 & 0,00 & - & 2,13 & 0,44 & 0,02 \\
\hline LD & 0,00 & 0,00 & - & 0,00 & 0,00 & - & 4,26 & 1,77 & 0,13 \\
\hline CFC & 12,00 & 2,35 & 0,39 & 15,00 & 14,14 & 4,42 & 29,79 & 14,49 & 7,79 \\
\hline RF & 0,00 & 0,00 & - & 2,50 & 0,66 & $\mathbf{0 , 0 3}$ & 0,00 & 0,00 & - \\
\hline MV & 60,00 & 15,14 & 12,75 & 17,50 & 4,28 & 1,56 & 51,06 & 10,62 & 8,81 \\
\hline TOTAL & - & 100,00 & 100,00 & - & 100,00 & 100,00 & - & 100,00 & 100,00 \\
\hline
\end{tabular}

A análise de agrupamento indicou que o outono e o inverno foram as estações com maior similaridade nos valores de $I A$, próxima a $90 \%$, e em conjunto com o verão formam agrupamento com similaridade próxima a $85 \%$, devido a maior participação de vegetais neste período em relação à primavera. Assim, considerandose valores de similaridade acima de $85 \%$, primavera foi a estação isolada das demais, principalmente pelo consumo quase exclusivo de Ostracoda e Tanaidacea, que juntos correspondem a valores de $I A$ superiores a $94 \%$ nas três áreas, sendo sempre superiores a $64 \%$ nas demais estações, fato que confere similaridade próxima a $70 \%$ para todo o conjunto (Figura 4 ).

Igualmente, diferenças espaciais foram registradas. A riqueza de itens na dieta foi maior nos rios que na Baía, salvo no verão, época em que apenas no São João o número de itens foi superior ao da Baía. Exceto pelas Larvas de Decapoda, os itens registrados no Cubatão o foram também no São João, e dois deles (Amphipoda e Cirripedia) não foram registrados na Baía. Mesmo assim, a análise de agrupamento indicou que Cubatão e Baía apresentaram similaridade superior a $80 \%$, sendo São João a área isolada na análise (Figura 5), principalmente devido ao maior consumo de Ostracoda neste grupo em relação ao São João, item cujo valor de $I A$ foi sempre superior a 1 nas três áreas: a 40 no Cubatão, a 20 na Baía e a quatro no São João. Em Frequência de Ocorrência vários itens alcançaram valor igual ou superior a 50 , com destaque para Ostracoda e Tanaidacea no São João (todas as estações), Gastropoda na Baía e no Cubatão, e material vegetal nos dois rios (Tabela 6).

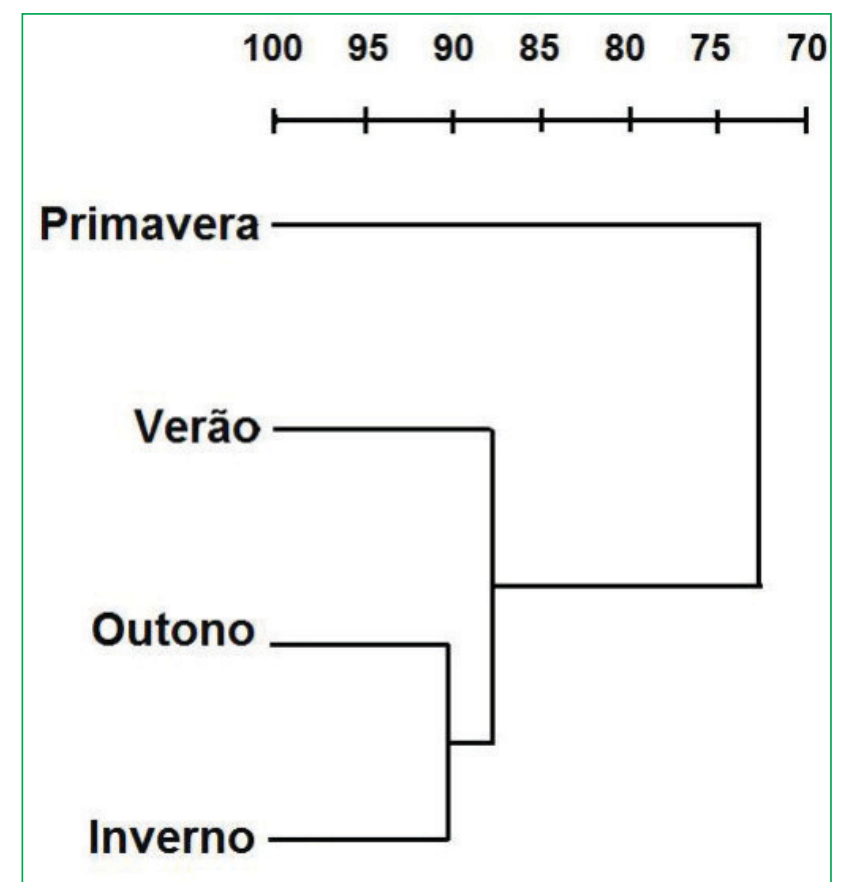

FIGURA 4: Análise de agrupamento dos valores de similaridade de Bray-Curtis do Índice Alimentar $(I A)$ da dieta de $C$. shufeldti entre as estações. 


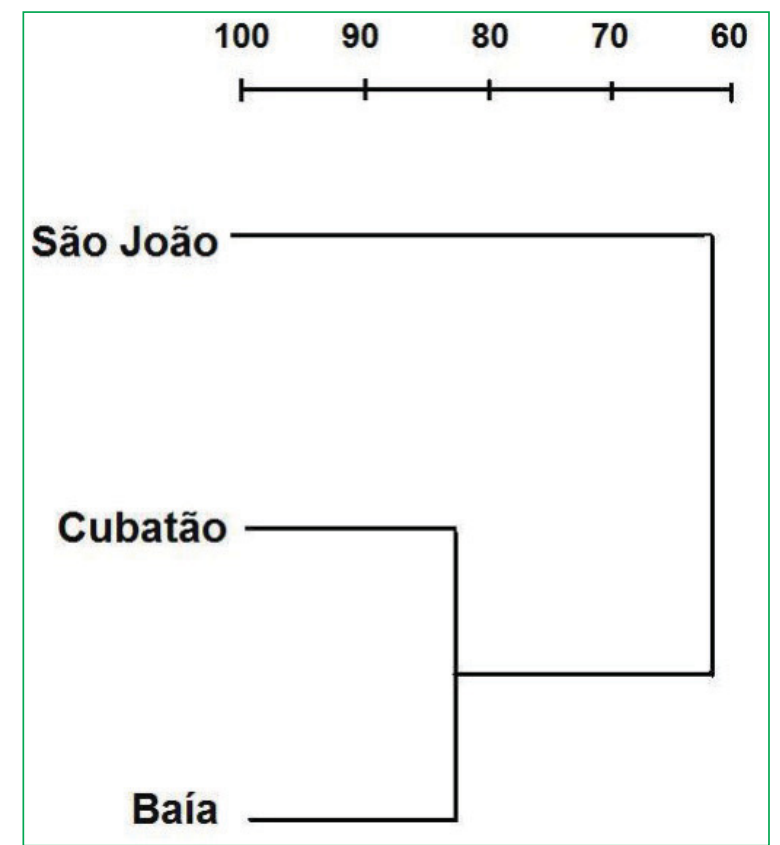

FIGURA 5: Análise de agrupamento dos valores de similaridade de Bray-Curtis do Índice Alimentar $(I A)$ da dieta de $C$. shufeldti entre as áreas São João, Cubatão e Baía.

TABELA 6: Itens do conteúdo estomacal de C. shufeldti cujos valores de Frequência de Ocorrência foram $\geq 50$, segundo estação do ano e área de coleta.

\begin{tabular}{|c|c|c|c|}
\hline & Cubatão & Baía & São João \\
\hline Primavera & $\begin{array}{l}\text { Ostracoda } \\
\text { Tanaidacea }\end{array}$ & Ostracoda & $\begin{array}{c}\text { Ostracoda } \\
\text { Tanaidacea }\end{array}$ \\
\hline Verão & Ostracoda & Ostracoda & $\begin{array}{l}\text { Ostracoda } \\
\text { Tanaidacea }\end{array}$ \\
\hline Outono & $\begin{array}{l}\text { Ostracoda } \\
\text { Gastropoda }\end{array}$ & $\begin{array}{c}\text { Ostracoda } \\
\text { Tanaidacea }\end{array}$ & $\begin{array}{c}\text { Ostracoda } \\
\text { Tanaidacea }\end{array}$ \\
\hline Inverno & $\begin{array}{c}\text { Ostracoda } \\
\text { Tanaidacea } \\
\text { Material vegetal }\end{array}$ & $\begin{array}{c}\text { Ostracoda } \\
\text { Gastropoda }\end{array}$ & $\begin{array}{c}\text { Ostracoda } \\
\text { Tanaidacea } \\
\text { Material } \\
\text { vegetal }\end{array}$ \\
\hline
\end{tabular}

\section{Discussão}

Dentre os seis grupos tróficos reconhecidos na Baía de Guaratuba por Chaves e Bouchereau (2004), C. shufeldti enquadra-se nos onívoros, o mesmo reconhecido por Caberty et al. (2004) em duas espécies de Gobiidae (Gobionellus oceanicus (Pallas, 1770) e Lophogobius cyprinoides (Pallas, 1770) em um estuário nas Antilhas. De fato, predação sobre invertebrados é comum na família (ex.: BOUCHEREAU et al., 1991; JOYEUX et al., 1991; ELGUEZABAL et al., 2003), e nesta espécie, em particular, consumo de crustáceos bentônicos (notadamente Ostracoda e Tanaidacea) já fora noticiado por Corrêa e Uieda (2007) e Contente (2008) em estuários próximos à Baía de Guaratuba. $\mathrm{O}$ último autor assinalou Ostracoda Cytherideidae e o Tanaidacea Kalliapseudes schubarti. Entretanto, o presente estudo mostra que a dieta compõe-se também de algas e outros vegetais, uma variação das situações relatadas por Depczynski e Bellwood (2003) quanto à participação de peixes criptobênticos na teia trófica. Os comportamentos então descritos em recifes de coral foram de peixes criptobênticos que ou predavam sobre invertebrados de fundo, ou predavam sobre invertebrados e também ingeriam detritos. Em Guaratuba, C. shufeldti enquadrase numa terceira possibilidade, aquela em que à ingestão de invertebrados soma-se a de algas e vegetais, ou seja, onivoria. É provável que esta seja um hábito comum em peixes de áreas rasas da Baía de Guaratuba (CHAVES; VENDEL, 2008) e ao qual Chaves e Bouchereau (2004) estimaram corresponder a maior biomassa de peixes dessa Baía, resulte da dupla influência (marinha e continental) a que está submetida a região de estudo (BOUCHEREAU; CHAVES, 2003). Trata-se de hábito alimentar também relatado por Caberty et al. (2004) em espécies de Gobiidae de manguezal nas Antilhas, mas não nos recifes de coral estudados por Depczynski e Bellwood (2003). Neste ambiente, as espécies de Gobiidae herbívoras não incluem em seus hábitos a predação sobre invertebrados.

Considerando que entre as três áreas e as quatro estações a diferença de tamanho dos espécimes foi de no máximo $30 \%$ entre os CT menores e $25 \%$ entre os CT maiores, é razoável assumir-se que o tamanho dos espécimes não comprometa interpretações sobre variações espaço-temporais da dieta. Padrão semelhante foi encontrado por Contente (2008), que registrou valores de similaridade superiores a $70 \%$ para espécimes com CT $\geq 21 \mathrm{~mm}$. A influência espacial manifestou-se não apenas pela maior variedade de itens e ocorrência de vegetais nos espécimes dos rios que nos da Baía, mas também pela distinção de dietas entre São João e Cubatão. É possível que tal distinção entre dois rios vizinhos esteja associada às diferenças na vegetação marginal entre as áreas, conforme proposto por Chaves e Vendel (2008) a partir de dados 
sobre a dieta de peixes de marismas e de manguezal nessa mesma região. Quanto às variações temporais, se por um lado a primavera foi a estação com maior grau de distinção das demais, por outro o verão destacou-se por um consumo expressivamente menor de vegetais. Isso surpreende, pois verão é a época em que a Baía recebe um aumento no aporte fluvial, consequentes da descarga promovida pelas chuvas (BOUCHEREAU; CHAVES, 2003). Tal aporte pode elevar a abundância de vegetais no sistema e, pelo aumento da turbidez na água, reduzir a detecção visual de invertebrados pelos peixes (CURIO, 1976). Mas na região os registros são variados quanto à participação de vegetais na dieta de peixes: enquanto no bagre Genidens genidens a abundância é maior no verão (CHAVES; VENDEL, 1996), no cangulo Stellifer rastrifer (CHAVES; VENDEL, 1998) ela o é no inverno. É possível, então, que a maior participação vegetal na dieta de $C$. shufeldti no outono e inverno resulte não do presumido aumento na disponibilidade desse item, mas, sim, de uma menor disponibilidade de invertebrados nessas estações. É o caso dos cirripédios, registrados apenas no inverno, época de maior influência oceânica na região (BOUCHEREAU; CHAVES, 2003), o que favorece a penetração de crustáceos marinhos na Baía. Tal flexibilidade de consumo é comum em peixes de manguezal, mas parece ser ainda mais importante nas espécies residentes quando comparado às migradoras (CABERTY et al., 2004; CHAVES; BOUCHEREAU, 2004).

\section{Agradecimentos}

Trabalho financiado pelo CNPq, Edital CT-Hidro/ CNPq 44/2006, processo 555483/2006-1.

\section{Referências}

BOUCHEREAU, J. L.; CHAVES, P. T. Ichthyofauna in the ecological organisation of a south-west Atlantic mangrove ecosystem: the bay of Guaratuba, south east Brazil. Vie et Milieu, Banyuls-sur-Mer, v. 53, n. 2-3, p. 103-110, 2003.

CABERTY, S.; BOUCHEREAU, J. L.; CHAVES, P. T. Organisation et fonctionnement trophiques de l'assemblage ichtyque d'um écosystème lagunaire à mangrove antillais au moyen de l'indice trophique de contribution. Cahiers de Biologie Marine, Rennes, v. 45, p. 243-254, 2004.

CURIO, E. The ethology of predation. Berlin : Springer-Verlag, 1976. $250 \mathrm{p}$.
CHAVES, P. T.; VENDEL, A. L. Aspectos da alimentação de Genidens genidens (Valenciennes) (Siluriformes: Ariidae) na Baía de Guaratuba, Paraná. Revista Brasileira de Zoologia, v. 13, n. 3, p. 669-675, 1996.

CHAVES, P. T.; CORRÊA, M. F. M. Composição Ictiofaunística da área de manguezal da Baía de Guaratuba, Paraná, Brasil. Revista Brasileira de Zoologia, Curitiba, v. 15, n. 1, p. 195-202, 1998.

CHAVES, P. T.; VENDEL A. L. Hábitos alimentares de Stellifer rastrifer (Perciformes: Sciaenidae) no manguezal de Guaratuba, Paraná, Brasil. Brazilian Archives of Biology and Technology, Curitiba, v. 41, n. 4, p. 423-428, 1998.

CHAVES, P. T.; VENDEL, A. L. Nota complementar sobre a composição ictiofaunística da Baía de Guaratuba, Paraná, Brasil. Revista Brasileira de Zoologia, Curitiba, v. 18, n. suplemento 1, p. 349-352, 2001.

CHAVES, P. T.; BOUCHEREAU, J. L. Trophic organization and functioning of fish populations in the Bay of Guaratuba, Brazil, on the basis of a trophic contribution factor. Acta Adriatica, Split, v. 45, n. 1, p. 83-94, 2004.

CHAVES, P.; VENDEL, A. L. Análise comparativa da alimentação de peixes (Teleostei) entre ambientes de marisma e de manguezal num estuário do sul do Brasil (Baía de Guaratuba, Paraná). Revista Brasileira de Zoologia, Curitiba, v. 25, p. 10-15, 2008.

CONTENTE, R. F. Partição inter-específica e efeitos sazonais, espaciais e ontogenéticos no uso de recursos tróficos por seis Teleostei em um sistema estuarino sub-tropical. 2008. $180 \mathrm{f}$. Dissertação (Mestrado em Zoologia) - Universidade Federal do Paraná, Curitiba. 2008.

CORRÊA, M. O. D. A.; UIEDA, V. S. Diet of the ichthyofauna associated with marginal vegetation of a mangrove forest in southeastern Brazil. Iheringia - Série Zoologia, Porto Alegre, v. 97, n. 4, p. 486-497, 2007.

DEPCZYNSKI, M.; BELLWOOD, D. R. The role of cryptobenthic reef fishes in coral reef trophodynamics. Marine Ecology Progress Series, Oldendorf/Luhe v. 256, p. 18-191, 2003.

ELGUEZABAL, E. M.; RUIZ, L.; TORRES, A.; RIVAS, A.; MARTINEZ, L. Hábitos alimentarios de Coryphopterus glaucofraenum (Pisces: Gobiidae) en la Bahia de Mochima, Estado Sucre, Venezuela. Ciência, Maracaibo, v. 11, n. 1, p. 31-38, 2003.

FIGUEIREDO, J. L.; MENEZES, N. A. Manual de peixes marinhos do sudeste do Brasil. Vol. 4. São Paulo: Museu de Zoologia da Universidade de São Paulo, 1985. 105 p.

FROESE, R.; PAULY, D. (Ed.). FISHBASE. 2010. Disponível em <http://fishbase.org/Summary/FamilySummary.php?ID=405>. versão 05/2010. Acesso em: 16 julho 2010.

JOYEUX, J. C.; TOMASINI, J. A.; BOUCHEREAU, J. L. Le régime alimentaire de Gobius niger Linné, 1758 (Teleostei, Gobiidae) dans la lagune de Mauguio - France. Annales des Sciences Naturalles Zoologie et Biologia Animale, Paris, v. 12, p. 57-69, 1991.

KAWAKAMI, E.; VAZZOLER, G. Método gráfico e estimativo do índice alimentar aplicado no estudo de alimentação de peixes. Boletim do Instituto Oceanográfico, São Paulo, v. 29, n. 2, p. $205-$ 207, 1980.

NELSON, J. S. Fishes of the World. 4 ed. New York: John Wiley \& Sons, 2006. $624 \mathrm{p}$.

PATZNER, R. A. Habitat utilization and depth distribution of small cryptobenthic fishes (Blenniidae, Gobiesocidae, Gobiidae, Tripterygiidae) in Ibiza (western Mediterranean Sea). Environmental Biology of Fishes, New York, v. 55, p. 207-214, 1999. 